
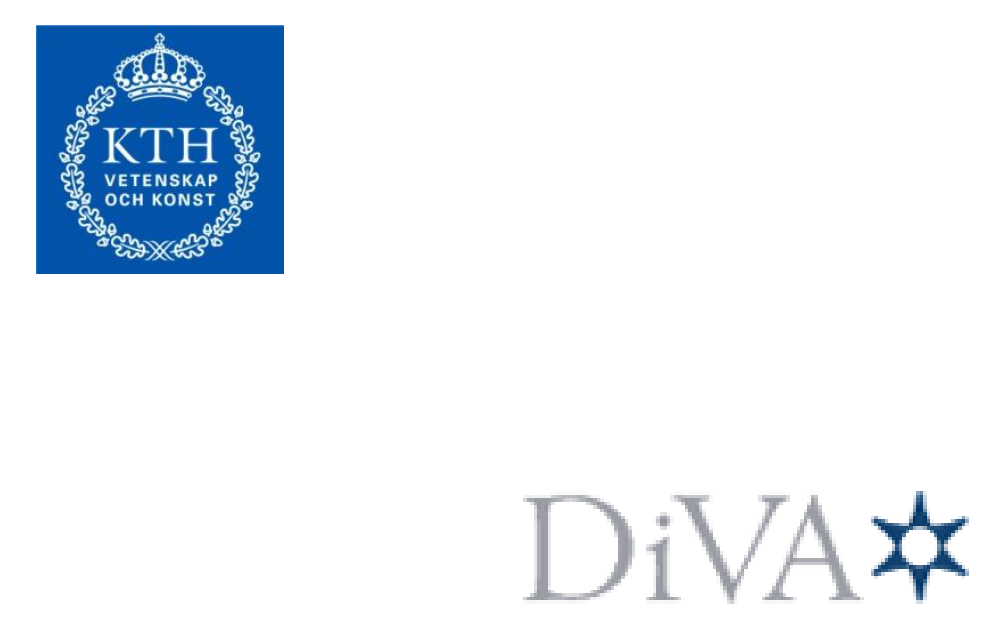

http://kth.diva-portal.org

This is an author produced version of a paper published in IEEE Transactions on Parallel and Distributed Systems.

This paper has been peer-reviewed but does not include the final publisher proofcorrections or proceedings pagination.

(C) 2013 IEEE. Personal use of this material is permitted. Permission from IEEE must be obtained for all other uses, in any current or future media, including reprinting/republishing this material for advertising or promotional purposes, creating new collective works, for resale or redistribution to servers or lists, or reuse of any copyrighted component of this work in other works.

Citation for the published paper:

Pangun Park, Piergiuseppe Di Marco, Carlo Fischione and Karl Henrik Johansson. Modeling and optimization of the IEEE 802.15.4 protocol for reliable and timely communications.

IEEE Transactions on Parallel and Distributed Systems.

Access to the published version may require subscription.

Published with permission from: IEEE 


\title{
Modeling and Optimization of the IEEE 802.15.4 Protocol for Reliable and Timely Communications
}

\author{
Pangun Park, Piergiuseppe Di Marco, Carlo Fischione, Karl Henrik Johansson
}

\begin{abstract}
Distributed processing through ad hoc and sensor networks is having a major impact on scale and applications of computing. The creation of new cyber-physical services based on wireless sensor devices relies heavily on how well communication protocols can be adapted and optimized to meet quality constraints under limited energy resources. The IEEE 802.15.4 medium access control protocol for wireless sensor networks can support energy efficient, reliable, and timely packet transmission by a parallel and distributed tuning of the medium access control parameters. Such a tuning is difficult, because simple and accurate models of the influence of these parameters on the probability of successful packet transmission, packet delay, and energy consumption are not available. Moreover, it is not clear how to adapt the parameters to the changes of the network and traffic regimes by algorithms that can run on resource-constrained devices. In this paper, a Markov chain is proposed to model these relations by simple expressions without giving up the accuracy. In contrast to previous work, the presence of limited number of retransmissions, acknowledgments, unsaturated traffic, packet size, and packet copying delay due to hardware limitations is accounted for. The model is then used to derive a distributed adaptive algorithm for minimizing the power consumption while guaranteeing a given successful packet reception probability and delay constraints in the packet transmission. The algorithm does not require any modification of the IEEE 802.15 .4 medium access control and can be easily implemented on network devices. The algorithm has been experimentally implemented and evaluated on a test-bed with off-the-shelf wireless sensor devices. Experimental results show that the analysis is accurate, that the proposed algorithm satisfies reliability and delay constraints, and that the approach reduces the energy consumption of the network under both stationary and transient conditions. Specifically, even if the number of devices and traffic configuration change sharply, the proposed parallel and distributed algorithm allows the system to operate close to its optimal state by estimating the busy channel and channel access probabilities. Furthermore, results indicate that the protocol reacts promptly to errors in the estimation of the number of devices and in the traffic load that can appear due to device mobility. It is also shown that the effect of imperfect channel and carrier sensing on system performance heavily depends on the traffic load and limited range of the protocol parameters.
\end{abstract}

Index Terms-IEEE 802.15.4, Markov chain model, Optimization.

\section{INTRODUCTION}

Wireless sensor and actuator networks have a tremendous potential to improve the efficiency of many distributed systems, for instance, in building automation and process control [1], [2]. Unfortunately, the current technology does not offer guaranteed energy efficiency and stability for closed-loop systems due to the packet losses and varying delays of the network. These systems are particularly challenging because they must support the right decision at the right moment by parallel and distributed algorithms despite traffic conditions, unexpected congestion, network failures, or external manipulations of the environment. The packet losses and delays are a very important quality measurement since it influences performance and stability of closed-loop systems [2]. Starting from these requirements, the development of efficient and reliable systems based on wireless sensor devices relies heavily on how well communication protocols can be adapted and optimized to

The authors are with the ACCESS Linnaeus Center, Electrical Engineering, Royal Institute of Technology, Stockholm, Sweden. E-mails: \{pgpark, pidm, carlofi,kallej\}@ee.kth.se.

This work was supported by the EU project FeedNetBack, the Swedish Research Council, the Swedish Strategic Research Foundation, the Swedish Governmental Agency for Innovation System, and Knut and Alice Wallenberg Foundation. meet quality constraints under limited energy resources.

The IEEE 802.15.4 standard has received considerable attention as a low data rate and low power protocol for wireless sensor network (WSN) applications in industry, control, home automation, health care, and smart grids [3]. Many of these applications require that packets are received with a given probability of success (reliability). In addition to such a reliability constraint, other applications ask for timely packet delivery [2]. However, since high reliability and low delay may demand a significant energy consumption of the network, thus reducing the WSN lifetime, the reliability and delay must be flexible design parameters that need to be adequate for the application requirements. It is known that IEEE 802.15.4 may have poor performance in terms of power consumption, reliability and delay [4], unless the medium access control (MAC) parameters are properly selected. A star network is an important topology for a number of standardization groups [5] and commercial products [6] such as asset tracking, process control, and building automation. Note that even such a simple topology presents highly challenging dynamics to model. It is essential to improve existing models of IEEE 802.15.4 in order to (a) characterize the protocol performance limitations, and (b) to tune the IEEE 802.15.4 parameters to enhance network lifetime and improve the quality of the service. 
This paper focuses on the modeling and optimization of the performance metrics (reliability, delay, power consumption) for IEEE 802.15.4 WSNs. This problem is specially appealing for many control and industrial applications [2]. We show that existing analytical studies of IEEE 802.15.4 are not adequate to capture the real-world protocol behavior, when there are retry limits to send packets, acknowledgements (ACKs), unsaturated traffic, and hardware limitations. We derive and use an extended model to pose an optimization problem where the objective function is the power consumption of the devices, the constraints are the reliability and delay of the packet delivery. The main idea of the proposed analysis is to tradeoff the power consumption of the network with the application requirements in terms of reliability and delay.

The remainder of this paper is as follows. In Section 2, we summarize existing work of analytical modeling and adaptive tuning of IEEE 802.15.4. Section 3 lists the main contributions of the paper and their relation to the literature. In Section 4, we propose an extended Markov chain model of CSMA/CA mechanism. In Section 5, the optimization problem to adapt the MAC parameters is investigated. In addition, implementation issues are also discussed. Numerical results for both the analytical model and the adaptive algorithm are presented in Section 6. Finally, Section 7 concludes the paper.

\section{Related Work}

The modeling of IEEE 802.15.4 is related to IEEE 802.11 [7]. We first discuss the literature concerning the analysis of IEEE 802.11 and 802.15.4, then we review previous work about adaptive MAC mechanisms for these protocols.

\subsection{Analytical Model of MAC}

Both IEEE 802.11 and 802.15.4 are based on a MAC that uses a binary exponential backoff scheme. Bianchi's model describes the basic functionalities of the IEEE 802.11 through a Markov chain under saturated traffic condition [8]. Extensions of this model have been used to analyze the MAC layer service time [9] and throughput [10] of IEEE 802.11.

The analysis of the packet delay, throughput, and power consumption of IEEE 802.15.4 WSNs has been the focus of the simulations-based study [11], and some more recent analytical works, e.g., [4], [12]-[15]. Inspired by Bianchi's work, a Markov model for IEEE 802.15.4 and an extension with ACK mechanism have been proposed in [4]. A modified Markov model including retransmissions with finite retry limits has been studied in [12] as an attempt to model the slotted carrier sense multiple access with collision avoidance (CSMA/CA) mechanism. In [13], a throughput analysis has been performed by an extension of the Markov chain model proposed [14]. The superframe structure, ACK, and retransmissions have been considered. However, the proposed Markov chain does not model the length of data and ACK packets, which is crucial to analyze the performance metrics for IEEE 802.15.4 networks with low data rate. Furthermore, in [14], the power consumption, reliability, and delay performance have not been investigated. In [15], a query-based approach has been considered to analyze the throughput, reliability, and delay of the network. However, the ACK mechanism and retransmission mechanism have not been accounted for.

The renewal theory has been applied to analyze the performance of IEEE 802.15.4 protocol in [16], [17]. In [16], the model of the slotted CSMA/CA based on the renewal process has been proposed and validated through Monte Carlo simulations in terms of throughput and service time. A contraction mapping technique is used to solve numerically the analytical equation, which is similar to the one of the Markov chain model in [8]. The contraction mapping approach is feasible for this model because the ACK mechanism and retransmission have not been considered. The beacon-enabled mode considering both contention access mechanism and inactive period of IEEE 802.15.4 protocol has been modelled using the renewal theory in [17]. Similar to the previous study [16], the ACK mechanism and the retransmission have not been accounted for. Even though the CSMA/CA algorithm of the beaconenabled mode explicitly uses the double carrier sensing in the standard, the single carrier sensing is only considered in [17].

We remark here that the analytical models available from the literature use numerical methods [4], [12]-[15] or a contraction mapping technique [16], [17] to solve nonlinear equations, which is a major drawback for in-network processing [18]. The computation complexity and computing time of optimization tools in WSNs are critical factors since the typical micro-controller does not support well a heavy computing. Typical sensors use $8 \mathrm{MHz}$ TI MSP 430 microcontroller [19]. In [20], the complex interdependence of the decision variables leads to difficult problems even in simple network topologies, where the analytical relations describing reliability, delay and energy consumption may be highly nonlinear expressions. Practical hardware limitations are not accurately considered in [4], [12]- [17]. Furthermore, despite the theoretical promises of the analytical models for analyzing the IEEE 802.15.4 MAC using Monte Carlo simulations, these theoretical results have not been validated by experiments.

\subsection{Adaptive Tuning of MAC}

Several algorithms to tune the MAC of IEEE 802.11 and IEEE 802.15.4 protocols have been proposed. The algorithms can be grouped in those based on the use of physical layer measurements, and those based on the use of link-layer information.

An adaptive tuning based on physical layer measurements has been investigated in [21]- [23], where a $p$-persistent IEEE 802.11 protocol has been considered to optimize the average backoff window size. The channel access probability $p$ that maximizes the throughput or minimizes the power consumption has been derived. This algorithm and its scalability to the network size have been studied also for IEEE 802.15.4 [23]. However, the channel sensing mechanism, the optional ACK, and retransmission mechanisms therein proposed are hard to be approximated by a $p$-persistent MAC. Furthermore, in [22] and [23] a saturated traffic regime has been assumed, which is a scenario of reduced interest for typical WSN applications. In [24], a cross-layer MAC design has been proposed by estimating both the MAC queue dynamics of each traffic class and the overall network contention level to meet the requirement of delay sensitive applications. However, this 
approach is not energy efficient because each device requires to monitor the queue dynamics and contention level.

Link-based optimizations for IEEE 802.11 have been investigated in [25], [26], where simple window adjustment mechanisms that are based on ACK transmissions have been considered. In these papers, the algorithms adapt the contention window size depending on the successful packet transmission, packet collision and channel sensing state, but the algorithms are not grounded on an analytical study. In [27], the authors have proposed an algorithm to tune the contenting window based on the multivariable control theory. Unfortunately, an IEEE 802.15.4 enhancement based on the use of link-layer information has the drawback of requiring a modification of the standard, and of a costly ACK mechanism since it introduces large overhead for small packets, although linkbased mechanisms are simple to implement. For instance, alarm messages in industrial control application are a single byte whereas the ACK has a size of 11 byte. In addition, the ACK mechanism requires extra waiting time. Link-based algorithms adapt the MAC parameters for each received ACK, which mean a slow and inefficient adaptation to network, traffic, and channel variations.

For IEEE 802.15.4 enhancements, there are less researches than on IEEE 802.11. Many results mainly focus on the energy efficiency of the devices rather than on explicit consideration of application requirements. In [28], a topology control algorithm has been proposed to reduce the average duty cycle of the network by reducing the number of coordinators without explicit consideration of packet reception rate or delay requirements. By considering the event sensing capability, a duty cycle algorithm is proposed for the beacon-enabled mode of the IEEE 802.15.4 protocol in [29]. Bernoulli scheduling where each device serves the packet with probability $P_{\text {ber }}$ or starts the sleep state with probability $1-P_{\text {ber }}$ is used for the energy management. This algorithm requires a modification of the standard. The analytical model uses the probability generating function (PGF) to describe the probability distribution of the packet service time at the MAC layer. However, the evaluation of PGF requires heavy computations.

\section{Original Contribution}

We consider a network with $N$ devices transmitting toward the personal area network coordinator (PAN). The devices use the beacon-enabled slotted CSMA/CA and ACK. The parameters of the CSMA/CA algorithm that influence reliability, delay and energy consumption are the minimum value of the backoff exponent macMinBE, the maximum number of backoffs before declaring a channel access failure macMaxCSMABackoffs, and the maximum number of retries allowed after a transmission failure macMaxFrameRetries that each device can select.

In this paper, we propose a novel modeling and adaptive tuning of the IEEE 802.15.4 MAC for reliable and timely communication while minimizing the energy consumption. The protocol is adjusted dynamically by a constrained optimization problem that each device of the network solves. The objective function, denoted by $\widetilde{E}_{\text {tot }}$, is the total energy consumption for transmitting and receiving packets of a device. The constraints are given by the reliability and average delay. The constrained optimization problem for a transmitting device is

$$
\begin{array}{cl}
\min _{\mathbf{V}} & \widetilde{E}_{\text {tot }}(\mathbf{V}) \\
\text { s.t. } & \widetilde{R}(\mathbf{V}) \geq R_{\min } \\
& \widetilde{D}(\mathbf{V}) \leq D_{\max } \\
& \mathbf{V}_{0} \leq \mathbf{V} \leq \mathbf{V}_{m}
\end{array}
$$

The decision variables of the device $\mathbf{V}=\left(m_{0}, m, n\right)$ are $m_{0} \triangleq \operatorname{macMinBE}, m \triangleq \operatorname{macMaxCSMABackoffs}, n \triangleq$ macMaxFrameRetries. $\widetilde{R}(\mathbf{V})$ is the reliability, and $R_{\min }$ is the minimum desired probability for successful packet delivery. $\widetilde{D}(\mathbf{V})$ is the average delay for a successfully received packet, and $D_{\max }$ is the desired maximum average delay. The constraint $\mathbf{V}_{0} \leq \mathbf{V} \leq \mathbf{V}_{m}$ captures the limited range of the MAC parameters. In the problem, we used the symbol $\sim$ to evidence that the energy, reliability, and delay expression are approximations. We will show later that we use approximations of high accuracy and reduced computational complexity so that devices can solve the problem.

Main contributions of the paper are the following: (a) the modeling of the relation between the MAC parameters of IEEE 802.15.4 and the selected performance metrics, (b) the derivation of simple relations to characterize the operations of the MAC by computationally affordable algorithms, (c) formulation and solution of a novel optimization problem for the MAC parameters, (d) the practical implementation of the optimization by a distributed adaptive algorithm on a test-bed using TelosB sensors [19]. and (e) performance evaluations of the algorithm by experiments and simulations of both stationary and transient network conditions.

The numerical evaluation of the performance metrics asks in general for heavy computations. This is a drawback when using them to optimize the IEEE 802.15.4 MAC parameters by in-network processing [18], because a complex computation is out of reach for resource limited sensing devices. Unlike previous work, we devise a simplified and effective method that reduces drastically the computational complexity while ensuring a satisfactory accuracy.

Based on our modeling, the adaptive IEEE 802.15.4 is furnished with two distinctive features: it does not require any modification of the existing standard, and it makes an optimization of all the MAC parameters of IEEE 802.15.4. Specifically, in contrast to link-based adaptation, our algorithm does not require ACK mechanism or request to send/clear to send (RTS/CTS) handshakes. In contrast to [21] - [23], we do not use the (inaccurate) $p$-persistent approximation and the modification of the standard therein proposed, and we do not require any hardware modification to make an estimate of the signal-to-noise ratio. Our adaptive tuning optimizes the considered MAC parameters, all at once, and not only some of them, as proposed in [21]-[27].

Our previous work [30] has proposed an adaptive algorithm for minimizing the power consumption while guaranteeing reliability and delay constraints in the packet transmission based on a Markov chain model [31]. In this paper, we extend this model to include the packet copying delay due to hardware 


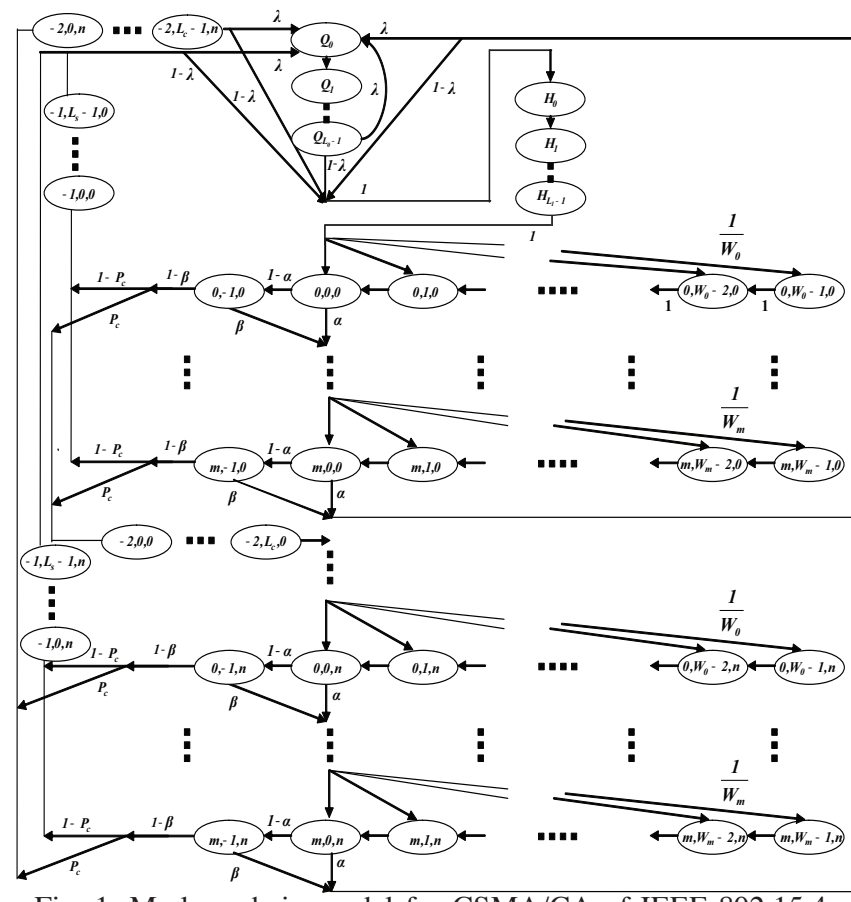

Fig. 1: Markov chain model for CSMA/CA of IEEE 802.15.4.

limitations and the imperfect channel. The computation time for certain performance metrics by solving nonlinear equations and by using an approximated approach is compared. We implement the IEEE 802.15.4 protocol to demonstrate the feasibility of the analytical model and to analyze the performance of the adaptive algorithm in a practical system. Furthermore, we show the effect of imperfect channel and carrier sensing failures on system performance through analysis and simulation results.

\section{Analytical Modeling}

In a star network, all $N$ devices contend to send data to the PAN coordinator, which is the data sink. Throughout this paper we consider applications where devices asynchronously generate packets with probability $1-\lambda$ when a device sends a packet successfully or discard a packet or the sampling interval is expired. Otherwise a device stays for $L_{0} S_{b}$ s without generating packets with probability $\lambda$, where $L_{0}$ is an integer and $S_{b}$ is the time unit aUnitBackoffPeriod (corresponding to 20 symbols). The data packet transmission is successful if an ACK packet is received.

In such a scenario, we propose an effective analytical model of the slotted CSMA/CA by a Markov chain. The chain gives us the objective function, energy (1a), and constraints on reliability (1b) and delay (1c) of the optimization problem. Experimental results validate the proposed model.

\subsection{Markov Chain Model}

In this section, we develop a Markov chain model of the slotted CSMA/CA mechanism of beacon-enabled IEEE 802.15.4.

Let $s(t), c(t)$ and $r(t)$ be the stochastic processes representing the backoff stage, the state of the backoff counter, and the state of the retransmission counter at time $t$ experienced by a device to transmit a packet. The triple $(s(t), c(t), r(t))$ is the three-dimensional Markov chain in Fig. 1, where we use $(i, k, j)$ to denote a particular state. The state of the retransmission counter is used to discard a packet if the transmission fails after $n+1$ attempts. Such a three-dimensional chain can be converted into a two-dimensional Markov chain if the MAC parameters $m_{0}, m, m_{b}$ of the CSMA/CA algorithm are initialized in each retransmission. We remark that the three-dimensional Markov chain model proposed in this paper is more general than a two-dimensional Markov chain because it allows the CSMA/CA algorithm to depend on the packet retransmissions. By assuming independent probability that devices start sensing, the stationary probability $\tau$ that a device attempts a first carrier sensing in a randomly chosen time slot is constant and independent of other devices. We denote the MAC parameters by $\mathbf{V}=\left(m_{0}, m, n\right), m_{b} \triangleq$ $\operatorname{macMaxBE}, W_{0} \triangleq 2^{m_{0}}, W_{m} \triangleq 2^{\min \left(m_{0}+m, m_{b}\right)}$.

The Markov chain consists of five main parts corresponding to the idle-queue states, packet copying states, backoff states, clear channel assessment (CCA) states, and packet transmission states. The states $\left(Q_{0}, \ldots, Q_{L_{0}-1}\right)$ correspond to the idlequeue states when the packet queue is empty and the device is waiting for the next packet generation time. Hence, the device sets its radio to sleep mode during the idle-queue states. Note that the idle-queue states $\left(Q_{0}, \ldots, Q_{L_{0}-1}\right)$ take into account the sampling interval. The states $\left(H_{0}, \ldots, H_{L_{1}-1}\right)$ represent the packet copying delay between the microcontroller and the radio transceiver, where

$$
L_{1}=\left\lceil\frac{L}{\nu S_{b}}\right\rceil,
$$

with $L$ being the total length of a packet including overhead and payload and $\nu$ is the serial peripheral interface (SPI) bus speed. Note that before sending a packet, the microcontroller copies the packet data into the transmit buffer of the radio transceiver over the SPI bus. In [32], the authors show that the packet copying is a very critical issue when forwarding a packet. The states from $\left(i, W_{m}-1, j\right)$ to $\left(i, W_{0}-1, j\right)$ represent the backoff states. The radio circuits of the device is set in idle mode or in sleep mode during the backoff period. The states $(i, 0, j)$ and $(i,-1, j)$ represent first $\mathrm{CCA}\left(\mathrm{CCA}_{1}\right)$ and second CCA $\left(\mathrm{CCA}_{2}\right)$, respectively. Let $\alpha$ be the probability that $\mathrm{CCA}_{1}$ is busy, and $\beta$ the probability that $\mathrm{CCA}_{2}$ is busy. The states $(-1, k, j)$ and $(-2, k, j)$ correspond to the successful transmission and packet collision, respectively. By knowing the duration of an ACK frame, ACK timeout, interframe spacing (IFS), data packet length, and header duration, we define the packet successful transmission time $L_{s}$ and the packet collision time $L_{c}$ as

$$
\begin{aligned}
& L_{s}=L+t_{\mathrm{ack}}+L_{\mathrm{ack}}+I F S, \\
& L_{c}=L+t_{\mathrm{m}, \mathrm{ack}},
\end{aligned}
$$

where $t_{\text {ack }}$ is ACK waiting time, $L_{\text {ack }}$ is the length of ACK frame, and $t_{\mathrm{m}, \text { ack }}$ is the timeout of the ACK, see details in [3].

We have the following results:

Proposition 1: Let the stationary probability of the Markov chain in Fig. 1 be

$$
b_{i, k, j}=\lim _{t \rightarrow \infty} P(s(t)=i, c(t)=k, r(t)=j),
$$


where $i \in(-2, m), k \in\left(-1, \max \left(W_{i}-1, L_{s}-1, L_{c}-1\right)\right), j \in$ $(0, n)$. Then, for $0 \leq i \leq m$

$$
b_{i, k, j}=\frac{W_{i}-k}{W_{i}} b_{i, 0, j}, \quad 0 \leq k \leq W_{i}-1,
$$

where

$$
W_{i}=\left\{\begin{array}{l}
2^{i} W_{0}, \quad i \leq m_{b}-m_{0}, \\
2^{m_{b}}, \quad i>m_{b}-m_{0},
\end{array}\right.
$$

and

$$
b_{i, 0, j}=\left((1-x) P_{c} \sum_{i=0}^{m} x^{i}\right)^{j} x^{i} b_{0,0,0},
$$

where $x=\alpha+(1-\alpha) \beta$,

$$
\begin{aligned}
& b_{0,0,0}=
\end{aligned}
$$

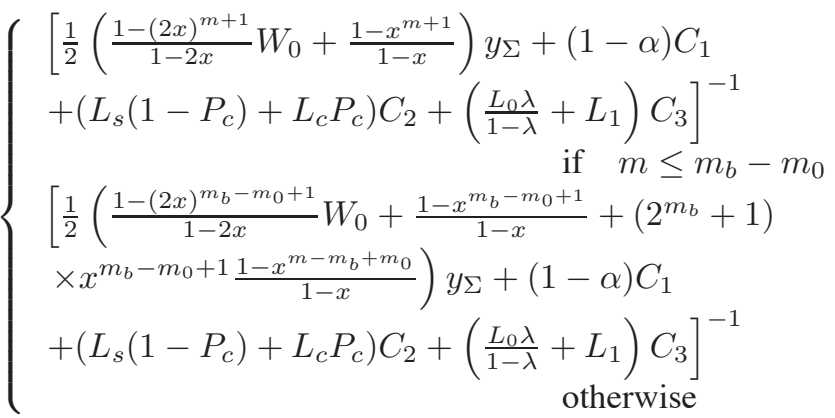

where $C_{1}=\frac{1-x^{m+1}}{1-x} y_{\Sigma}, C_{2}=\left(1-x^{m+1}\right) y_{\Sigma}, C_{3}=$ $\left(\left(1-P_{c}\right)\left(1-x^{m+1}\right)+x^{m+1}\right) y_{\Sigma}+P_{c}\left(1-x^{m+1}\right) y^{n}, y_{\Sigma}=$ $\frac{1-y^{n+1}}{1-y}, y=P_{c}\left(1-x^{m+1}\right)$, and $P_{c}$ is the collision probability. Moreover,

$$
b_{-1, k, j}=\left(1-P_{c}\right)(1-x) \sum_{i=1}^{m} b_{i, 0, j}, \quad 0 \leq k \leq L_{s}-1,
$$

and

$$
b_{-2, k, j}=P_{c}(1-x) \sum_{i=1}^{m} b_{i, 0, j}, \quad 0 \leq k \leq L_{c}-1 .
$$

Proof: See Section A of [33].

We remark here that the term $b_{0,0,0}$, which plays a key role in the analysis, is different from the corresponding term given in [4], [12]- [15] due to our accurate modeling of the retransmissions, ACK, unsaturated traffic, packet size, and packet copying delay. In the next section, we demonstrate the validity of the Markov chain model by experiments.

Now, starting from Proposition 1, we derive the channel sensing probability $\tau$ and the busy channel probabilities $\alpha$ and $\beta$. The probability $\tau$ that a device attempts $\mathrm{CCA}_{1}$ in a randomly chosen time slot is

$$
\tau=\sum_{i=0}^{m} \sum_{j=0}^{n} b_{i, 0, j}=\frac{1-x^{m+1}}{1-x} \frac{1-y^{n+1}}{1-y} b_{0,0,0} .
$$

This probability depends on the probability $P_{c}$ that a transmitted packet encounters a collision, and the probabilities $\alpha$ and $\beta$. These probabilities are developed in the following.

Let $P_{c}$ be the retransmission probability. If the packet collides due to other transmitted packets or is lost due to a bad channel, then the device repeats the CSMA/CA algorithm until a maximum number of retransmissions $n$. We model the bad channel by independent Bernoulli trials with the bad channel probability $p$ where $0 \leq p \leq 1$. The independence of the trial results is assumed to be over all links and time slots. We assume that a transmitted packet in the bad channel does not affect other transmitted packets in the good channel. The slow variation of a fading channel is assumed so that the received power is constant throughout an entire transmission. The packets are discarded due to either of two reasons: (i) collision, or (ii) bad channel. A transmitted packet in the good channel collides if at least one of the $N-1$ remaining devices transmits in the good channel and in same time slot. In addition, a transmitted packet is lost due to the bad channel. If all devices transmit with probability $\tau, P_{c}$ is

$$
P_{c}=\left(1-(1-\tau(1-p))^{N-1}\right)(1-p)+p,
$$

where $N$ is the number of devices. Similarly to [4], we derive the busy channel probabilities $\alpha$ and $\beta$ as follows:

$$
\alpha=\alpha_{1}+\alpha_{2},
$$

where $\alpha_{1}$ is the probability of finding channel busy during $\mathrm{CCA}_{1}$ due to data transmission, namely,

$$
\alpha_{1}=L\left(1-(1-\tau(1-p))^{N-1}\right)(1-\alpha)(1-\beta),
$$

and $\alpha_{2}$ is the probability of finding the channel busy during $\mathrm{CCA}_{1}$ due to ACK transmission, which is

$$
\begin{aligned}
\alpha_{2}= & L_{\text {ack }} \frac{N \tau(1-p)(1-\tau(1-p))^{N-1}}{1-(1-\tau)^{N}} \\
& \times\left(1-(1-\tau(1-p))^{N-1}\right)(1-\alpha)(1-\beta),
\end{aligned}
$$

where $L_{\text {ack }}$ is the length of the ACK. By a similar argument, the probability of finding the channel busy during $\mathrm{CCA}_{2}$ is

$$
\beta=\frac{1-(1-\tau)^{N-1}+N \tau(1-p)(1-\tau(1-p))^{N-1}}{2-(1-\tau)^{N}+N(1-p) \tau(1-\tau(1-p))^{N-1}} .
$$

Now, we are in the position to derive the carrier sensing probability $\tau$ and the busy channel probabilities $\alpha$ and $\beta$ by solving the system of non-linear equations (8), (9), and (10) for these probabilities, see details in [31]. From these probabilities then one could derive the expressions of the reliability, delay for successful packet delivery, and power consumption that are needed in (1). The drawback of such an approach is that there is no closed form expression for these probabilities, and the system of equations that gives $\tau, \alpha$ and $\beta$ must be solved by numerical methods. This may be computationally demanding and therefore inadequate for use in simple sensor devices. In the following, we instead present a simple analytical model of the reliability, delay for successful packet delivery, and power consumption. The key idea is that sensor devices can estimate the busy channel probabilities $\alpha$ and $\beta$ and the channel sensing probability $\tau$. Therefore, devices exploit local measurements to evaluate the performance metrics, rather than solving nonlinear equations. We compare the computation time to solve nonlinear equations and to use our proposed method in Section 6.2. Details follow in the sequel, where we derive these approximations for Eqs. (1a)-(1c). 


\subsection{Reliability}

The main contributions of this section are the derivation of both precise and approximated expression of the reliability (1b) of the optimization problem (1), where we recall the reliability is the probability of successful packet reception.

In slotted CSMA/CA, packets are unsuccessfully received due to two reasons: channel access failure and retry limits. Channel access failure happens when a packet fails to obtain idle channel in two consecutive CCAs within $m+1$ backoffs. Furthermore, a packet is discarded if the transmission fails due to repeated collisions after $n+1$ attempts. Following the Markov model presented in Fig. 1, the probability that the packet is discarded due to channel access failure is

$$
P_{d c}=x^{m+1} \sum_{j=0}^{n} y^{j}=\frac{x^{m+1}\left(1-y^{n+1}\right)}{1-y} .
$$

The probability of a packet being discarded due to retry limits is

$$
P_{d r}=y^{n+1} .
$$

Therefore, the reliability is

$$
R(\mathbf{V})=1-\frac{x^{m+1}\left(1-y^{n+1}\right)}{1-y}-y^{n+1} .
$$

Now, we derive an approximation of the reliability.

Approximation 1: An approximation of the reliability is

$$
\widetilde{R}(\mathbf{V})=1-x^{m+1}(1+\widetilde{y})-\widetilde{y}^{n+1}
$$

where

$$
\begin{aligned}
\widetilde{y}= & \left(1-\left(1-(1+x)(1+\hat{y}) \widetilde{b}_{0,0,0}\right)^{N-1}\right)\left(1-x^{2}\right), \\
\widetilde{b}_{0,0,0}= & 2 /\left(W_{0}(1+2 x)(1+\hat{y})+2 L_{s}\left(1-x^{2}\right)(1+\hat{y})\right. \\
& \left.+\left(L_{0} \lambda /(1-\lambda)+L_{1}\right)\left(1+\hat{y}^{2}+\hat{y}^{n+1}\right)\right),
\end{aligned}
$$

and $\hat{y}=\left(1-(1-\tau)^{N-1}\right)\left(1-x^{2}\right)$.

Derivation: See Section B of [33].

We remark that $\widetilde{R}(\mathbf{V})$ is a function of the measurable busy channel probabilities $\alpha$ and $\beta$, the channel access probability $\tau$, and the MAC parameters $m_{0}, m_{b}, m, n$. Note that $\alpha, \beta$, and $\tau$ are explicit functions of the number of devices and traffic load. Our basic idea is to use the measured busy channel probabilities and channel access probability to estimate the performance metrics, as well as to detect the network change. When the number of devices or the traffic load changes, it affects $\alpha, \beta$, and $\tau$.

\subsection{Delay}

In this section, we derive the constraint of average delay (1c) of the optimization problem (1). The average delay for a successfully received packet is defined as the time interval from the instant the packet is at the head of its MAC queue and ready to be transmitted, until the transmission is successful and the ACK is received. We develop an approximation for an average delay, which is given by Approximation 2. To this aim, we need some intermediate technical steps. In particular, we characterize (a) the expression of the delay for a successful transmission at time $j+1$ after $j$ th events of unsuccessful transmission due to collision and (b) the expected value of the approximated backoff delay due to busy channel.

Let $D_{j}$ be the random time associated to the successful transmission of a packet at the $j$ th backoff stage. Denote with $\mathscr{A}_{j}$ the event of a successful transmission at time $j+1$ after $j$ unsuccessful attempts. Let $\mathscr{A}_{t}$ be the event of successful transmission within the total attempts $n$. Then, the delay for a successful transmission after $j$ unsuccessful attempts is

$$
D=\sum_{j=0}^{n} \mathbb{1}_{\mathscr{A}_{j} \mid \mathscr{A}_{t}} D_{j}
$$

where $\mathbb{1}_{\mathscr{A}_{j} \mid \mathscr{A}_{t}}$ is 1 if $\mathscr{A}_{j} \mid \mathscr{A}_{t}$ holds, and 0 otherwise and $D_{j}=$ $L_{s}+j L_{c}+\sum_{h=0}^{j} T_{h}$, with $T_{h}$ being the backoff stage delay, $L_{s}$ is the packet successful transmission time, and $L_{c}$ is the packet collision transmission time as defined in Eq. (2).

A transmission is successful with probability $1-P_{c}$, or collide with probability $P_{c}$. The probability of the event is

$$
\operatorname{Pr}\left(\mathscr{A}_{j} \mid \mathscr{A}_{t}\right)=\frac{P_{c}^{j}\left(1-x^{m+1}\right)^{j}}{\sum_{k=0}^{n}\left(P_{c}\left(1-x^{m+1}\right)\right)^{k}}
$$

where the normalization comes by considering all the possible events of successful attempts $\mathscr{A}_{t}$. Therefore, the probability of successful transmission at time $j+1$ after $j$ events of unsuccessful transmission due to collision is

$$
\operatorname{Pr}\left(\mathscr{A}_{j} \mid \mathscr{A}_{t}\right)=\frac{(1-y) y^{j}}{1-y^{n+1}}
$$

In the following, we give the total backoff delay $T_{h}$. Let $T_{h, i}$ be the random time needed to obtain two successful CCAs from the selected backoff counter value in backoff level $i$. Denote with $\mathscr{B}_{i}$ the event occurring when the channel is busy for $i$ times, and then idle at the time $i+1$. Let $\mathscr{B}_{t}$ be the event of having a successful sensing within the total number of $m$ sensing attempts. If the device accesses an idle channel after its $i$ th busy CCA, then $T_{h}=\sum_{i=0}^{m} \mathbb{1}_{\mathscr{B}_{i} \mid \mathscr{B}_{t}} T_{h, i}$, where

$$
T_{h, i}=2 T_{s c}+\sum_{k=1}^{i} T_{h, k}^{s c}+\sum_{k=0}^{i} T_{h, k}^{b}
$$

and where $2 T_{s c}$ is the successful sensing time, $\sum_{k=1}^{i} T_{h, k}^{s c}$ is the unsuccessful sensing time due to busy channel during CCA, and $\sum_{k=0}^{i} T_{h, k}^{b}$ is the backoff time.

By considering the busy channel during two CCAs, the probability of the event $\mathscr{B}_{i} \mid \mathscr{B}_{t}$ is approximated by

$$
\widetilde{\operatorname{Pr}}\left(\mathscr{B}_{i} \mid \mathscr{B}_{t}\right)=\frac{\gamma^{i}}{\sum_{k=0}^{m} \gamma^{k}},
$$

where $\gamma=\max (\alpha,(1-\alpha) \beta)$. Note that the approximation is based on the worst case of the busy channel probabilities. The approximation of the average backoff period is

$$
\begin{aligned}
\mathbb{E}\left[\widetilde{T}_{h}\right] & =\sum_{i=0}^{m} \widetilde{\operatorname{Pr}}\left(\mathscr{B}_{i} \mid \mathscr{B}_{t}\right) \mathbb{E}\left[\widetilde{T}_{h, i}\right] \\
& =2 T_{s c}+\sum_{i=0}^{m} \widetilde{\operatorname{Pr}}\left(\mathscr{B}_{i} \mid \mathscr{B}_{t}\right) \sum_{k=0}^{i}\left(\frac{2^{k} W_{0}-1}{2} S_{b}+2 T_{s c} k\right)
\end{aligned}
$$


where the approximated sensing time $\mathbb{E}\left[\widetilde{T}_{h, i}\right]$ considers the worst case, i.e., a failure of $\mathrm{CCA}_{2}$, which implies that $T_{s c}=$ $S_{b}$ and that each sensing failure takes $2 T_{s c}$. Therefore, the expected value of the approximated backoff delay is

$$
\begin{aligned}
\mathbb{E}\left[\widetilde{T}_{h}\right]= & 2 S_{b}\left(1+\frac{1}{4}\left(\frac { 1 - \gamma } { 1 - \gamma ^ { m + 1 } } \left(2 W_{0} \frac{1-(2 \gamma)^{m+1}}{1-2 \gamma}\right.\right.\right. \\
& \left.\left.\left.-\frac{3(m+1) \gamma^{m+1}}{1-\gamma}\right)+\frac{3 \gamma}{1-\gamma}-\left(W_{0}+1\right)\right)\right),
\end{aligned}
$$

where $\gamma=\max (\alpha,(1-\alpha) \beta)$.

Approximation 2: The expected value of the approximated delay is

$$
\begin{aligned}
\widetilde{D}(\mathbf{V})= & T_{s}+\mathbb{E}\left[\widetilde{T}_{h}\right] \\
& +\left(\frac{y}{1-y}-\frac{(n+1) y^{n+1}}{1-y^{n+1}}\right)\left(T_{c}+\mathbb{E}\left[\widetilde{T}_{h}\right]\right) .
\end{aligned}
$$

Derivation: By considering the Eq. (15), we derive

$$
\widetilde{D}(\mathbf{V})=\sum_{j=0}^{n} \operatorname{Pr}\left(\mathscr{A}_{j} \mid \mathscr{A}_{t}\right) \mathbb{E}\left[\widetilde{D}_{j}\right]
$$

where $\mathbb{E}\left[\widetilde{D}_{j}\right]=T_{s}+j T_{c}+\sum_{h=0}^{j} \mathbb{E}\left[\widetilde{T}_{h}\right]$ and $\mathbb{E}\left[\widetilde{T}_{h}\right]$ is given in Eq. (19).

We remark that the expected value of the approximated delay is an explicit function of the busy channel probabilities and channel access probability, whereas the approximation does not require explicit information of the number of devices and traffic load. In Section 6, we will show that the busy channel probabilities and channel access probabilities represent well the effect of the number of devices and traffic load also in practice.

\subsection{Power Consumption}

Here, we derive the objective function, power consumption of the device (1a) of the optimization problem (1). We propose two models for the average power consumption, depending on the radio state during the backoff mechanism specified by the IEEE 802.15.4 standard. Let us denote by I-mode and $S$ mode the situation when the radio is set in idle mode or in sleep mode during backoff period, respectively. The device sets its radio to sleep mode during the idle-queue states for $L_{0} S_{b}$ s. Note that since we consider the uplink communication from the devices to the coordinator, the energy consumption of CSMA/CA mechanism and traffic load are critical factors for the energy consumption of the devices.

Approximation 3: The energy consumption of the I-mode is

$$
\begin{aligned}
\widetilde{E}_{\mathrm{tot}, \mathrm{i}}(\mathbf{V})= & \frac{P_{i} \tau}{2}\left[\frac{(1-x)\left(1-(2 x)^{m+1}\right)}{(1-2 x)\left(1-x^{m+1}\right)} W_{0}-1\right] \\
& +P_{s c}(2-\alpha) \tau+C_{4}+P_{w}\left(x^{m+1}(1+y)\right. \\
& \left.+\left(P_{c} y^{n}+\left(1-P_{c}\right)(1+y)\right)\left(1-x^{2}\right)\right) \widetilde{b}_{0,0,0}
\end{aligned}
$$

and of the $S$-mode is

$$
\begin{aligned}
\widetilde{E}_{\mathrm{tot}, \mathrm{s}}(\mathbf{V})= & P_{w}\left(\tau-\frac{\widetilde{b}_{0,0,0}\left(1-(0.5 x)^{m+1}\right)}{W_{0}(1-0.5 x)} \frac{1-y^{n+1}}{1-y}\right) \\
& +P_{s c}(2-\alpha) \tau+C_{4}
\end{aligned}
$$

where state probability $\widetilde{b}_{0,0,0}$ is given in [33], $C_{4}=$ $(1-\alpha)(1-\beta) \tau\left(P_{t} L+P_{i}+L_{\text {ack }}\left(P_{r}\left(1-P_{c}\right)+P_{i} P_{c}\right)\right)$, $P_{i}, P_{s c}, P_{s p}, P_{w}, P_{t}$ and $P_{r}$ are the average power consumption in idle-listen, channel sensing, sleep states, wake-up state, transmit and receiving states, respectively.

Derivation: See Section C of [33].

\section{IEEE 802.15.4 OPTIMIZATION}

In the previous sections we developed the expressions of the performance metrics. Here, we present a novel approach where each device locally solves the optimization problem. Consider the reliability, delay and power consumption as investigated in Section 4. The optimization problem (1) can be written by using Eq. (14) given by Approximation 1 for reliability constraint, Eq. (20) given by Approximation 2 for delay constraint and Eq. (21) or (22) given by Approximation 3 for the power consumption. Note that the power consumption is given by Eq. (21) if the I-mode is selected, and it is given by Eq. (22), if the $S$-mode is selected. The solution of the optimization problem gives the optimal MAC parameter $\left(m_{0}, m, n\right)$ that each device uses to minimize its energy expenditure, subject to reliability and delay constraints. The problem is combinatorial because the decision variables take on discrete values.

A vector of decision variables $\mathbf{V}$ is feasible if the reliability and delay constraints are satisfied. The optimal solution may be obtained by checking every combination of the elements of $\mathbf{V}$ that gives feasibility, and then checking the combination that gives the minimum objective function. Clearly, this approach may have a high computational complexity, since there are $6 \times 4 \times 8=192$ combinations of MAC parameters to check [3]. Therefore, we propose an algorithm that gives the optimal solution by checking just a reduced number of combinations. From Figs. 2, 3 and 4, we remark here that the reliability and power consumption of both $I$-mode and $S$ mode are increasing function as the parameter $n$ increases. This property is quite useful to solve (1) by a simple algorithm with reduced computational complexity, as we see next.

The search of optimal MAC parameters uses an iterative procedure according to the component-based method [34]. In particular, the probabilities $\alpha, \beta$, and $\tau$ are estimated periodically by each device. If a device detects a change of these probabilities, then the device solves the local optimization problem (1) using these estimated values. The solution is achieved by finding the value of $n$ that minimizes the energy consumption given a pair of values for $m_{0}$ and $m$. Since the power consumption is increasing with $n$, it follows that the minimum is attained at the lowest value of $n$ that satisfies the constraints. Given that the reliability is increasing with $n$, simple algebraic passages give that such a value is $n=f\left(m_{0}, m\right)$, with

$$
f\left(m_{0}, m\right)=\left\lceil\frac{\ln \left(1-x^{m+1}(1+\widetilde{y})-R_{\min }\right)}{\ln (\widetilde{y})}-1\right\rceil,
$$

where $\widetilde{y}=\left(1-(1-\widetilde{\tau})^{N-1}\right)\left(1-x^{2}\right)$ and

$$
\widetilde{\tau}=\frac{2 r_{3}}{2^{m_{0}} r_{1}+2 r_{2}},
$$


with

$$
\begin{aligned}
& r_{1}=(1+2 x)(1+\hat{y}), \\
& r_{2}=L_{s}\left(1-x^{2}\right)(1+\hat{y})+\left(K_{0}+L_{1}\right)\left(1+\hat{y}^{2}+\hat{y}^{n+1}\right), \\
& r_{3}=(1+x)(1+\hat{y}),
\end{aligned}
$$

and $\hat{y}=\left(1-(1-\tau)^{N-1}\right)\left(1-x^{2}\right)$. Eq. (23) returns the optimal retry limits given a pair $m_{0}, m$. Notice that $x$ and $\hat{y}$ are measurable since device estimates $\alpha, \beta$, and $\tau$. By using this simple algorithm, a device checks just $6 \times 4=24$ combinations of the MAC parameters $m_{0}, m$ instead of $6 \times 4 \times 8=192$ combinations that would be required by an exhaustive search.

We propose an alternative approach having a low computation cost. The optimal solution $\left(m_{0}, m, n\right)$ of problem (1) can be computed off-line and stored in a look-up table as function of the busy channel probabilities $\alpha$ and $\beta$ and the channel access probability $\tau$. The table can be thought of as a matrix with the set of values of $\alpha, \beta, \tau$. Each device can estimate $\alpha, \beta, \tau$, and use $\left(m_{0}, m, n\right)$ from the look-up table the entries of the solutions at location $\alpha, \beta, \tau$ closer to the estimated values.

We have seen by the Approximations 1,2 and 3 that the performance metrics are function of the busy channel probabilities $\alpha$ and $\beta$ and the channel access probability $\tau$. Once these probabilities are known at a device, the optimal MAC parameters of that device can be readily computed by the simple algorithm. In the algorithm, the number of devices and packet generation rates are assumed to be known, whereas the busy channel probability and channel access probability are periodically estimated in each device during the sensing states of the MAC layer, and they do not require an ACK mechanism, as we describe the details in the following. In addition, the robustness of the algorithm to possible errors in the estimation of the number of devices and traffic load is then investigated in Section 6.3.2. In our experiment, we observe that the busy channel probabilities and channel access probability implicitly represent well the effect of the number of devices and traffic load even if we have erroneous estimation in terms of the number of devices and traffic load.

The average busy channel probabilities $\alpha$ and $\beta$ are estimated at each device while sending a data packet to the coordinator. The estimations of these probabilities simply use measured CCA information without any hardware modification. These probabilities are initialized at the beginning of the device's operation. The estimations of the busy channel probabilities and the channel access probability use a sliding window. When the device senses the channel at $\mathrm{CCA}_{1}$ or $\mathrm{CCA}_{2}$, these probabilities are updated by $\alpha=\delta_{b} \alpha+(1-$ $\left.\delta_{b}\right) \hat{\alpha}, \beta=\delta_{b} \beta+\left(1-\delta_{b}\right) \hat{\beta}$ for some $\delta_{b} \in(0,1)$, respectively. Note that $\hat{\alpha}$ and $\hat{\beta}$ are the busy channel probability of $\mathrm{CCA}_{1}$ and $\mathrm{CCA}_{2}$ of the current sliding window, respectively. Therefore, a device does not require any extra communication and sensing state to estimate these probabilities compared to the IEEE 802.15.4 standard. By contrast, the estimation algorithms for IEEE 802.11 proposed in [21] and [35] are not energy efficient since a device needs to sense the channel state during the backoff stage. This allows one to estimate the average length of idle period. Hence, these schemes are implementable only in I-mode. By contrast, our scheme is applied in both $I$ mode and $S$-mode and does not require any computation load during the backoff stage.

During an initialization phase of the algorithm, a device communicates with the initial MAC parameters $m_{0}=3, m_{b}=$ $8, m=4, n=1$. Then, the busy channel probabilities $\alpha$ and $\beta$ and the channel access probability $\tau$ are estimated in each device during the channel sensing state of IEEE 802.15.4 without any extra state. The application requirements are communicated by the coordinator to the device if there are changes. It is also possible that each device makes a decision of application requirements depending on the data type e.g., strict delay requirement for alarm message.

\section{Performance Evaluation}

We implement the IEEE 802.15.4 protocol to demonstrate the feasibility of the analytical model and to analyze the performance of the adaptive algorithm in a practical system. The IEEE 802.15.4 protocol was implemented on a test-bed using the TelosB platform [19] running the Contiki OS [36] based on the specifications of the IEEE 802.15.4 [3]. The IEEE 802.15.4 defines one backoff as 20 symbols that correspond to $320 \mu \mathrm{s}$ for $2.45 \mathrm{GHz}$. Since the hardware timer available for TelosB is based on a $32768 \mathrm{~Hz}$ clock, we use a backoff with duration of $305 \mu \mathrm{s}$ instead of the $320 \mu \mathrm{s}$. We consider a typical indoor environment with concrete walls. 10 devices are placed to mimic a star topology. Each device is at a distance of around $5 \mathrm{~m}$ from the coordinator. The implementation is available for download [37].

The packet generation time adopted in this paper varies depending on the backoff time of CSMA/CA and retransmission time described in Section 4, which is different from the typical periodic traffic pattern commonly assumed in WSNs. The network contention level of the periodic traffic model significantly depends on the initial packet generation time. Hence, the initial traffic generation time is a critical factor to analyze the different performance metrics such as reliability, delay, throughput, and energy consumption. Our traffic model explicitly considers the contention level of the network. The basic idea is that as the contention level increases, the packet generation time increases so that the collisions are reduced. On the other hand, as the contention level decreases, the packet generation time decreases to increase the throughput of the network. This simple model allows us to make a statistical analysis of the performance of the network. We remark that as the length of idle-queue increases, the proposed traffic model is similar to the periodic traffic model. The reason is that the length of idle-queue becomes dominant for packet generation interval rather than the packet delay.

In this section, we first validate the approximated model in terms of reliability, average delay, and energy consumption derived in Section 4 by experimental results. We compare the computation time for performance metrics by solving nonlinear equations or using an approximated model in Section 6.2. Then, we present an extensive set of experiments and Monte Carlo simulations to analyze the performance of the adaptive algorithm under both stationary and transient 


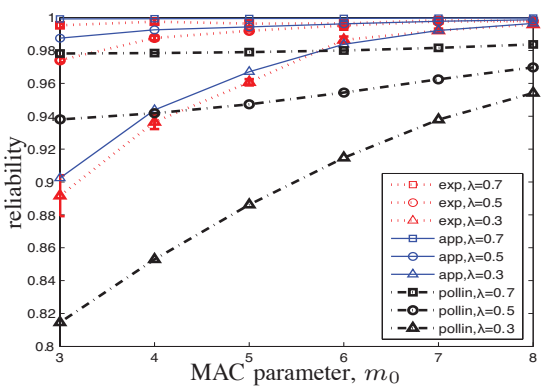

(a) $m_{0}=3, \ldots, 8, m_{b}=8, m=4, n=1$

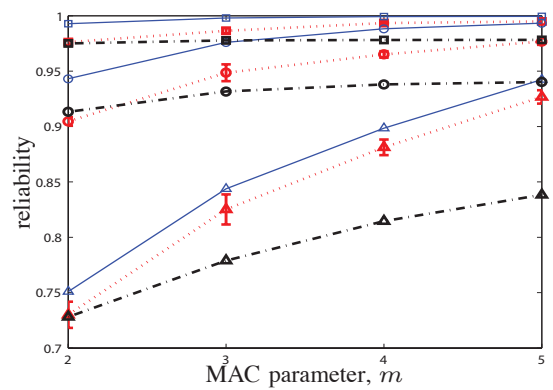

(b) $m=2, \ldots, 5, m_{0}=3, m_{b}=8, n=1$

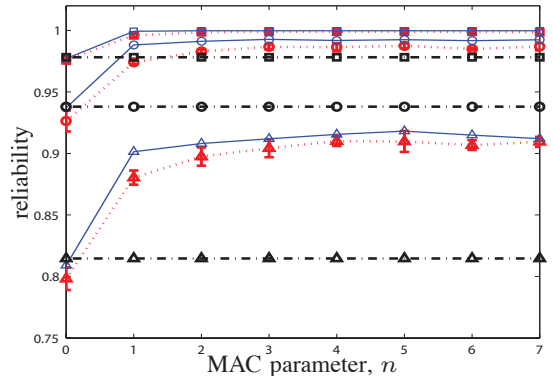

(c) $n=0, \ldots, 7, m_{0}=3, m_{b}=8, m=4$

Fig. 2: Reliability as a function of the traffic regimes $\lambda=0.3,0.5,0.7$, and MAC parameters $m_{0}=3, \ldots, 8, m_{b}=8, m=2, \ldots, 5$, $n=0, \ldots, 7$ as obtained by our proposed analysis, experimental implementation, and with Pollin's Markov chain model [4]. The length of the packet is $L=5$ and the number of devices is $N=10$. The vertical bars indicate the standard deviation as obtained out of 5 experimental runs of $2 \times 10^{5}$ time slots each. The percentage error of the analytical model for the reliability is $0.993 \%$.

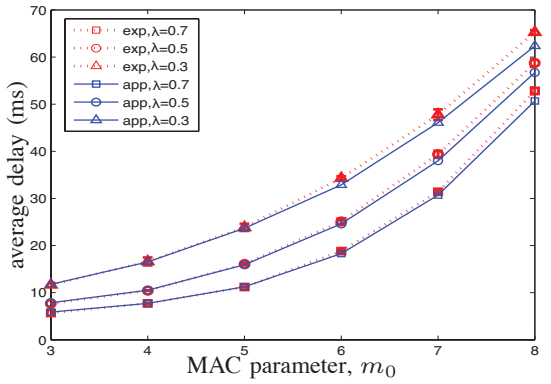

(a) $m_{0}=3, \ldots, 8, m_{b}=8, m=4, n=1$

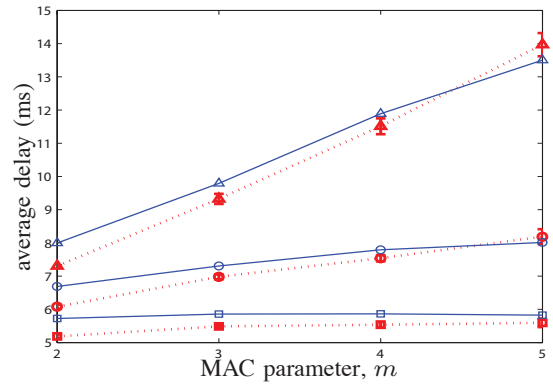

(b) $m=2, \ldots, 5, m_{0}=3, m_{b}=8, n=1$

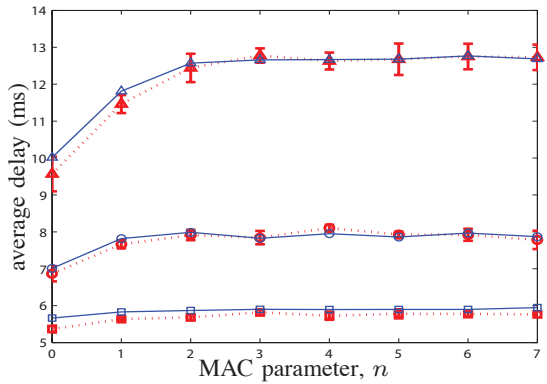

(c) $n=0, \ldots, 7, m_{0}=3, m_{b}=8, m=4$

Fig. 3: Average delay as a function of the traffic regimes $\lambda=0.3,0.5,0.7$ and MAC parameters $m_{0}=3, \ldots, 8, m_{b}=8, m=2, \ldots, 5$, $n=0, \ldots, 7$ as obtained by our proposed analysis and experimental implementation. The percentage error of the analytical model for the average delay is $3.155 \%$.

conditions. Furthermore, we discuss the effect of imperfect channel and carrier sensing capabilities by using simulations.

\subsection{Analytical Model Validation}

Fig. 2 compares the reliability given by Eq. (14), the analytical model in [4], and experimental results as a function of the traffic regimes $\lambda=0.3,0.5,0.7$ with $N=10$ devices and different MAC parameters $m_{0}, m, n$. The vertical bars indicate the standard deviation as obtained out of 5 experimental runs of $2 \times 10^{5}$ time slots each. In the figure, note that "Pollin" refers to the reliability model derived in [4]. Our analytical expression matches quite well the experimental results. Note that the percentage error of the reliability given by Eq. (14) is $0.993 \%$. The expression is closer to experimental results under low traffic regime $\lambda=0.5,0.7$ than high traffic regime $\lambda=0.3$ because the approximation holds if $x=\alpha+(1-\alpha) \beta \ll$ 1 , but $x$ increases as the traffic and the number of devices increases. The reliability approaches 1 under very low traffic regime $\lambda=0.7$. In Fig. 2(a), 2(b), the reliability increases as MAC parameters $m_{0}, m$ increase, respectively. In Fig. 2(c), we observe that the improvement of reliability is small as the retry limits $n$ increases for $n \geq 3$. Notice that the reliability saturates to 0.92 for traffic regime $\lambda=0.3$ for $n \geq 3$. The retransmissions are necessary but not sufficient to obtain high reliability under high traffic regimes.

Fig. 3 shows the average delay as obtained by Eq. (20) as a function of different traffic regimes $\lambda=0.3,0.5,0.7$ with a given number of devices $N=10$ and different MAC parameters $m_{0}, m, n$. The analytical model predicts well the experimental results. Note that the percentage error of the average delay given by Eq. (20) is $3.155 \%$. The accuracy is reduced under high traffic regime $\lambda=0.3$ due to the approximation. Observe that the average delay increases as traffic regime increases due to high busy channel probability and collision probability. Fig. 3(a) shows that the average delay increases exponentially as $m_{0}$ increases. Hence, we conclude that $m_{0}$ is the key parameter affecting the average delay when compared to $m, n$.

Fig. 4 compares our proposed analytical model and experimental results for the power consumption for both I-mode and $S$-mode as a function of different traffic regimes $\lambda=$ $0.3,0.5,0.7$ with a number of devices $N=10$ and different MAC parameters $m_{0}, m, n$. The percentage errors of the average power consumption for I-mode given by Eq. (21) and $S$ mode given by Eq. (22) are $0.193 \%$ and $0.175 \%$, respectively. The power consumption of $S$-mode and I-mode decreases as $m_{0}$ increases because of sleep mode and idle mode during the backoff time for high traffic regime $\lambda=0.3,0.5$. The main component of the average power consumption is the transmit or receiving power rather than power consumption during backoff time for high traffic regime, i.e., $P_{t}>P_{i}>P_{s p}$ and $P_{r}>P_{i}>P_{s p}$. However, the power consumption of $I$-mode increases as the MAC parameters $\left(m_{0}, m, n\right)$ increase under low traffic regime $\lambda=0.7$. Since the device needs to stay more time in idle sleep stage without packet generation, the main component of average power consumption is the idle backoff time rather than transmit or receiving power consumption under low traffic regime $\lambda=0.7$. Observe that the power 


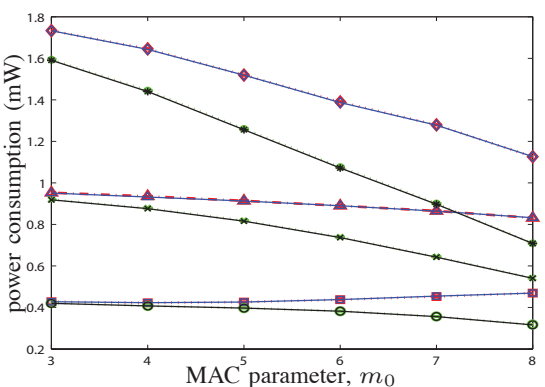

(a) $m_{0}=3, \ldots, 8, m_{b}=8, m=4, n=1$

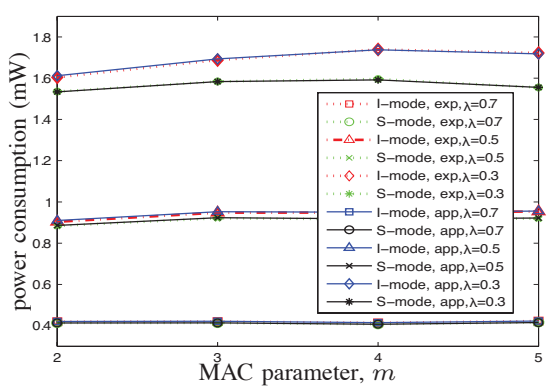

(b) $m=2, \ldots, 5, m_{0}=3, m_{b}=8, n=1$

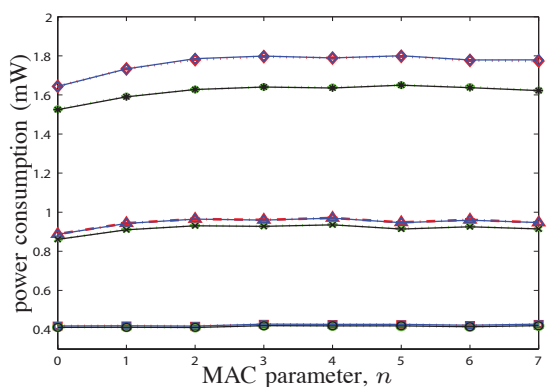

(c) $n=0, \ldots, 7, m_{0}=3, m_{b}=8, m=4$

Fig. 4: Average power consumption of I-mode and $S$-mode as a function of the traffic regimes $\lambda=0.3,0.5,0.7$ and MAC parameters $m_{0}=3, \ldots, 8, m_{b}=8, m=2, \ldots, 5, n=0, \ldots, 7$ as obtained by our analysis and experimental implementation. The percentage errors of the analytical model for the average power consumption are $0.193 \%$ and $0.175 \%$ for $I$-mode and $S$-mode, respectively.
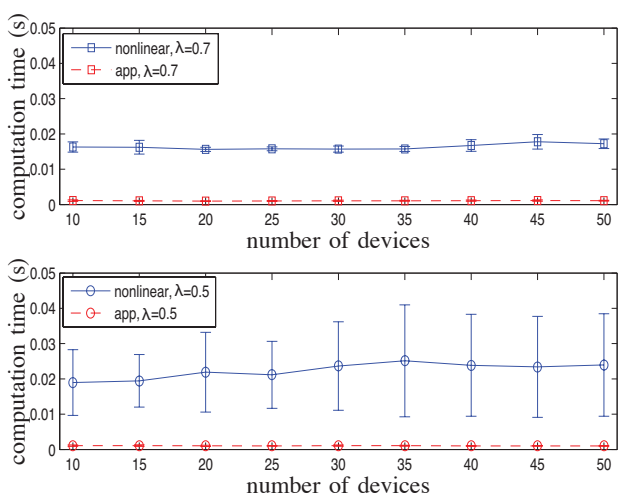

Fig. 5: Computation time for performance metrics by solving nonlinear equations and using an approximate approach as a function of the number of devices $N=10, \ldots, 50$, traffic regime $\lambda=0.5,0.7$. The vertical bars of solving nonlinear equations indicate the standard deviation as obtained out of 10 runs with different initial points of $\alpha, \beta, \tau$ between 0 and 0.5 .

consumption has a weaker dependence on $(m, n)$ than on $m_{0}$.

\subsection{Computation Time}

Fig. 5 compares the computation time for performance metrics including reliability, average delay, and energy consumption by solving nonlinear equations and using an approximate approach as a function of the number of devices $N=10, \ldots, 50$, traffic regime $\lambda=0.5,0.7$. The vertical bars indicate the standard deviation as obtained out of 10 runs with different initial points of $\alpha, \beta, \tau$ between 0 and 0.5 . The approximated model enables the computation time savings of $95 \%$ compared to the one of solving nonlinear equations. The system of nonlinear equations is solved using the common trust-region dogleg algorithm. When the traffic regime is high $\lambda=0.5$, the initial point is more critical for the computation time to apply this algorithm. The computation time by solving nonlinear equations shows weak dependency with the number of device and the traffic regime.

\subsection{Adaptive Algorithm}

In the following, we present an extensive set of real-world experiments and Monte Carlo simulations to analyze the performance of the new adaptive algorithm for tuning the MAC parameters, under both stationary and transient conditions. The analytical modeling that we have proposed in Section 4 is based on the Markov chain modeling that has been validated in Section 6.1. Hence, the Monte Carlo simulations are representative of the real-world behavior of the network.
In the stationary conditions, the application requirements and network scenario are constant, whereas in transient condition there are variations. In the experiments and simulations, the network considers the $I$-mode and $S$-mode of the device to compare the performance on the reliability, average packet delay and power consumption. Furthermore, we investigate the robustness to network changes and sensitivity to inaccurate parameter estimations. It is not possible to compare our algorithm to other algorithms from the literature as the link-based ones, because they modify the IEEE 802.15.4 standard and are focused on different performance metrics (e.g., throughput). However, it is possible to show that our algorithm outperform significantly the results in link-based ones. This is due to that these results use the ACK feedback, which has a low update frequency with respect to the channel and network variations, whereas our algorithm reacts much faster.

\subsubsection{Protocol Behavior in Stationary Conditions}

In this subsection, we investigate the improvement of performance metrics of the proposed scheme at stationary conditions of the network, namely without changing network scenarios.

Fig. 6 compares the experimental results for the reliability, average delay, and power gain values of the IEEE 802.15.4 MAC protocol as obtained by our algorithm and with default MAC parameters. The vertical bars indicate the standard deviation as obtained out of 5 experimental runs of $5 \times 10^{5}$ time slots each. Both the I-mode and $S$-mode for various traffic configurations and requirements are considered. The requirements for both the $I$-mode and $S$-mode are $R_{\min }=$ $0.9,0.95, D_{\max }=50$ and $R_{\min }=0.95, D_{\max }=20,100 \mathrm{~ms}$, respectively. Fig. 6(a) shows that both $I$-mode and $S$-mode satisfy the reliability constraint for different traffic regime. We observe a strong dependence of the reliability of default MAC on different traffic regime. At the high traffic regime $\lambda=0.2$, the reliability of default MAC is 0.89 . In Fig. 6(b), the delay constraint is fulfilled both I-mode and S-mode. Observe that average delay of I-mode decreases when traffic regime is low $\lambda \geq 0.4$. This is due to that the optimal MAC parameters at higher traffic regime increase more than the ones at lower traffic regime to satisfy the reliability constraint.

Recall that the target of our proposed adaptive algorithm is to use the tradeoff between application constraints and energy consumption instead of just maximization of reliability or minimization of delay. Therefore, to characterize quantita- 


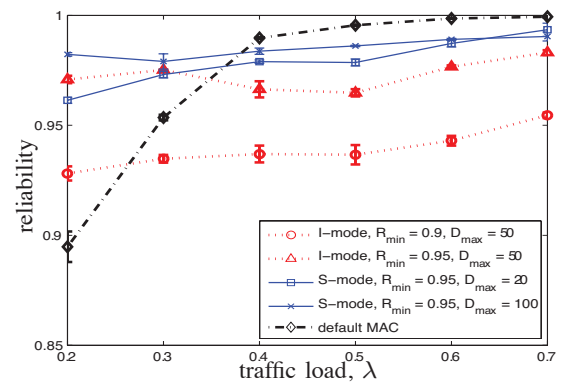

(a) Reliability

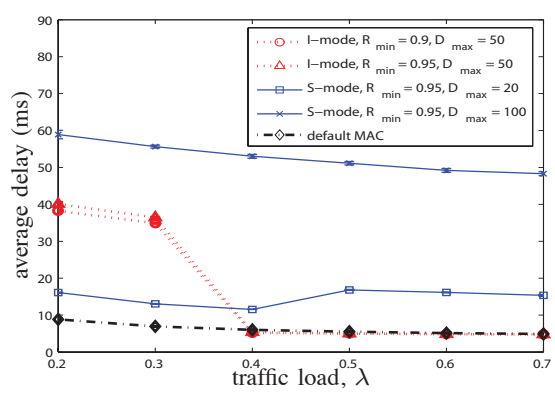

(b) Average delay

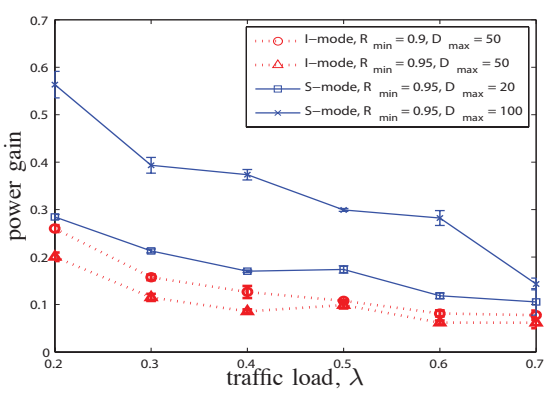

(c) Power gain

Fig. 6: Stationary condition in the experiment: reliability, average delay and power gain of the I-mode, S-mode of proposed scheme and IEEE 802.15.4 with default parameter $\left(m_{0}=3, m_{b}=5, m=4, n=3\right)$ as a function of the traffic load $\lambda=0.2, \ldots, 0.7$, the reliability requirement $R_{\min }=0.9,0.95$ and delay requirement $D_{\max }=20,50,100 \mathrm{~ms}$ for the length of the packet $L=5$ and $N=10$ devices. Note that "default MAC" refers to IEEE 802.15.4 with default MAC parameters. The vertical bars indicate the standard deviation as obtained out of 5 experimental runs of $5 \times 10^{5}$ time slots each.

tively the power consumption, we define the power gain as $\theta=\frac{E_{\mathrm{def}}-E_{\mathrm{tot}}(\mathbf{V})}{E_{\mathrm{def}}}$ where $E_{\mathrm{def}}$ and $E_{\mathrm{tot}}(\mathbf{V})$ are the average power consumption of I-mode or $S$-mode for default MAC and proposed scheme, respectively. The closer $\theta$ to 1 , the better the power efficiency. Fig. 6(c) shows that the power gain increases as traffic regime increases. This improvement is higher for $S$-mode than I-mode, e.g., $\theta \approx 0.57$ for $S$ mode with $R_{\min }=0.95, D_{\max }=100$. Although there is a strong dependence of the power gain on the traffic regime, our proposed algorithm gives a better energy efficiency than the default MAC. Therefore, the experimental results show clearly the effectiveness of our adaptive IEEE 802.15.4 protocol while guaranteeing the constraints.

\subsubsection{Robustness Analysis}

The adaptive IEEE 802.15 .4 protocol is based on the estimation of the busy channel probabilities $\alpha$ and $\beta$ and the channel access probability $\tau$. In this section, we investigate the convergence time of the optimal MAC parameters obtained by our distributed adaptive algorithm. The performance analysis carried out so far assumed that the number of devices and traffic configuration are fixed. One of the critical issues in the design of wireless networks is time varying condition. Therefore, we will investigate our algorithm to react to changes in the number of devices and traffic load when each device has an erroneous estimation of these parameters by using the Monte Carlo simulations.

Figs. 7(a), 7(b), 7(c) show the I-mode behavior of channel state, MAC parameters, and reliability of the Monte Carlo simulations when the number of devices changes from $N=10$ to $N=20$ with an erroneous estimation of the number of devices, respectively. At time $17.6 \mathrm{~s}$, the number of devices sharply increases to 20 , when it was estimated to be 10 . We assume that the wrong estimation happens due to some errors in the estimation phase or a biasing induced by the hiddennode phenomenon. This causes a significant increase of the contention level. Note that $n_{1}$ is one of existing devices before the network change and $n_{11}$ is one of the new devices that enters the network at time $17.6 \mathrm{~s}$.

In Fig 7(a), we observe that the busy channel probabilities $\alpha$ and $\beta$ and channel access probability $\tau$ of device $n_{11}$ become stable after the network changes by updating the
MAC parameters over time. In Section 5, we noticed that the update frequency of $\alpha, \beta, \tau$ is different. $\tau$ is updated in each aUnitBackoffPeriod and $\alpha$ and $\beta$ are updated when a device stay in $\mathrm{CCA}_{1}$ and $\mathrm{CCA}_{2}$, respectively. Hence, the update frequency order of $\alpha, \beta$, and $\tau$ is $\tau$ first, then $\alpha$, and finally $\beta$. We remark here that the update frequency of linkbased adaptation is lower than the update frequency of $\beta$ of our algorithm since link-based adaptation requires an ACK transmission. The update frequency of channel estimation is a critical issue where the traffic regime is low such as in monitoring applications.

Fig. 7(b) shows that the MAC parameters $\left(m_{0}, m, n\right)$ converge to $(3,2,0)$ of device $n_{1}$ and $n_{11}$. The figures indicate that the system reacts correctly to the erroneous estimation of the number of devices after a few seconds. We observe that the convergence of the MAC parameters of proposed scheme is very fast since our algorithm is based on analytical model instead of heuristic considerations as in link-based adaptation, where the algorithms adapt the contention window size by the ACK transmission. In addition, recall that our adaptive IEEE 802.15.4 is based on the carrier sensing information before transmitting packets. In Fig 7(c), the reliability fulfills the requirement $R_{\min }=0.95$ for both the existing and new devices. Note that the oscillation of reliability is due to packet loss. Similar behaviors are observed for $S$-mode.

Fig. 8 presents the behavior of the device when the traffic load changes sharply from $\lambda=0.8$ to $\lambda=0.5$ at time $25.6 \mathrm{~s}$. Devices use a wrong estimation of the traffic load, which is estimated to be $\lambda=0.8$, after the traffic load changes. The results indicate that our algorithm is quite effective for the traffic configuration change. In Fig. 8(a), the busy channel and channel access probability increase as a result of higher traffic regime $\lambda=0.5$ for both $I$-mode and $S$-mode. Fig. 8(b) shows that the parameter $m$ of $S$-mode updates from 2 to 5 due to the increasing busy channel probability after the traffic load changes at time $28 \mathrm{~s}$. The figure indicates that the system reacts correctly to the erroneous estimation of traffic configuration and, in few seconds, the estimation of $\alpha, \beta$ and $\tau$ allow to reach the optimal MAC parameters. Observe that the algorithm returns different parameters for $I$-mode and $S$-mode due to the different power consumption model. In Fig. 8(c), the reliability requirement $R_{\min }=0.95$ is fulfilled for both $I$-mode and $S$ - 


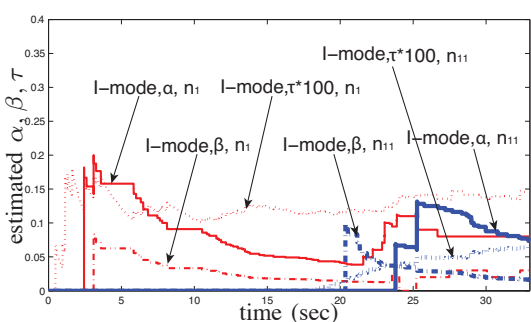

(a) $\alpha, \beta, \tau$ behavior

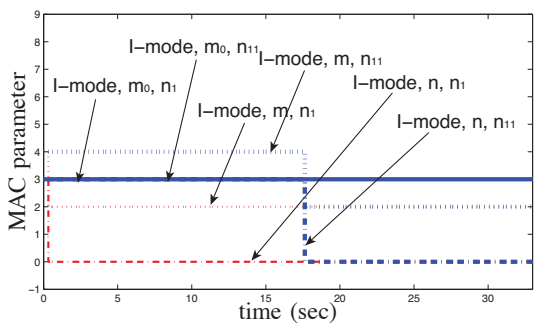

(b) MAC parameter $\left(m_{0}, m, n\right)$ behavior

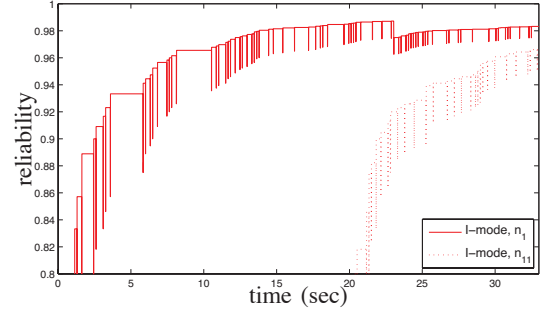

(c) Reliability behavior

Fig. 7: Robustness when the number of devices changes in the simulation: busy channel probabilities, channel access probability, MAC parameters and reliability behavior of I-mode when the number of devices changes sharply from $N=10$ to $N=20$ at time 17.6 s. Note that $n_{1}$ and $n_{11}$ represent the behavior of one of $N=10$ devices plus new devices after time $17.6 s$. Traffic load is $\lambda=0.6$, length of the packet is $L=3$, the reliability and delay constraint are $R_{\min }=0.95$ and $D_{\max }=100 \mathrm{~ms}$, respectively.

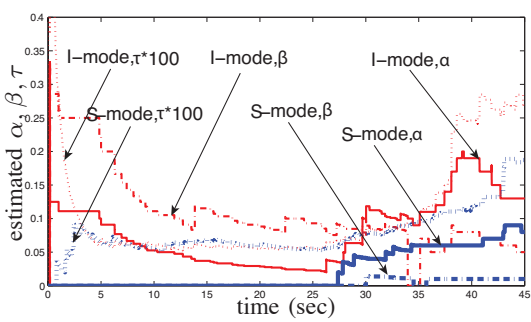

(a) $\alpha, \beta, \tau$ behavior

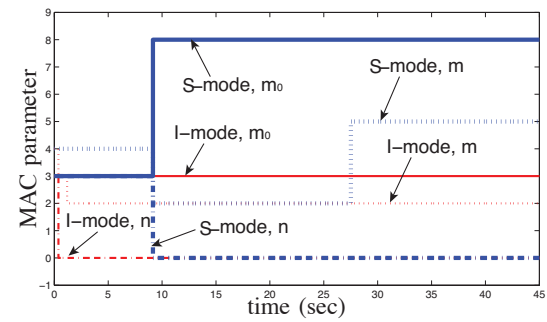

(b) MAC parameter $\left(m_{0}, m, n\right)$ behavior

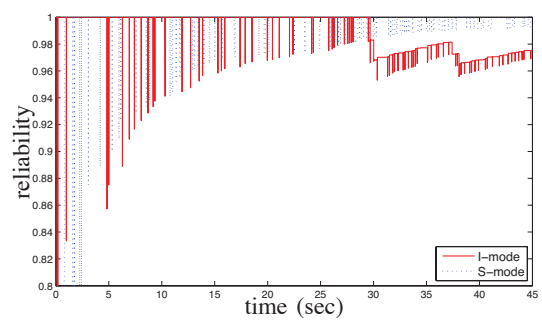

(c) Reliability behavior

Fig. 8: Robustness when the traffic load changes in the simulation: busy channel probabilities, channel access probability, MAC parameters, reliability and delay behavior of $I$-mode and $S$-mode when the traffic load changes sharply from $\lambda=0.8$ to $\lambda=0.5$ at time 25.6 s. The length of the packet is $L=3$, the reliability and delay constraint are $R_{\min }=0.95$ and $D_{\max }=100 \mathrm{~ms}$, respectively.

mode. The reliability of I-mode is greater than 0.95 with some fluctuations after traffic load increases.

\subsubsection{Sensitivity Analysis}

Even though the assumption of the perfect wireless channel and carrier sensing plays a critical role to understand the fundamental limitations of the IEEE 802.15.4 protocol, wireless channel and carrier sensing in practice are not perfect. In this section, we first analyze the effect of both imperfect wireless channel and imperfect carrier sensing through simulations. We also present the sensitivity of adaptive algorithm with respect to the estimation errors to the busy channel probabilities and the channel access probability. The main reason to use the simulation is that it is difficult to characterize the wireless channel and carrier sensing failure in real-world experiments.

Fig. 9 compares the reliability and average packet delay of the IEEE 802.15.4 protocol of our proposed model and simulation results as a function of the probabilities of the bad channel $p=0.1, \ldots, 0.5$, traffic regime $\lambda=0.1,0.4,0.8$ given MAC parameters $m_{0}=3, m=4, m_{b}=8, n=1$, the number of devices $N=10$, and packet length $L=7$. The effect of the imperfect wireless channel is modelled by a Bernoulli random process. The analytical model of the reliability as obtained by Eq. (13) predicts well the simulation results. The reliability decreases as the probability of bad channel increases due to the greater number of retransmissions. Each device retransmits a packet if an ACK is not received within the maximum number of retries. For the same reason, the average packet delay increases as the probability of the bad channel increases.

Now, we analyze the performance of the IEEE 802.15.4 protocol in the presence of carrier sensing errors. Two types of carrier sensing errors, i.e., false negative and false positive, are considered, and their impact on the system performance is

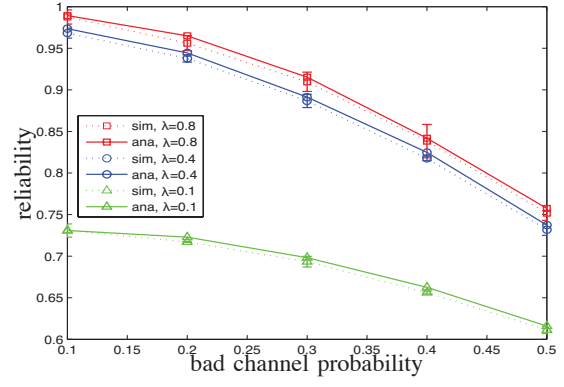

(a) Reliability

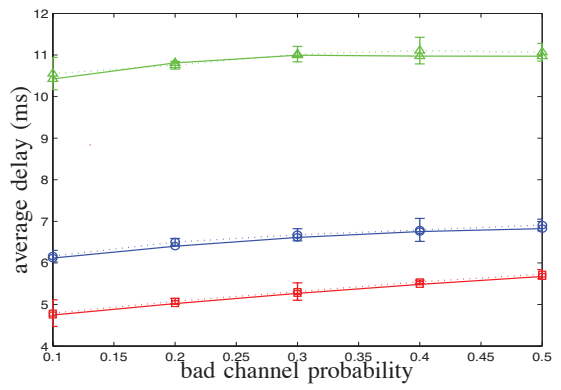

(b) Average delay

Fig. 9: Effect of imperfect channel as a function of loss probabilities $p=0.1, \ldots, 0.5$, traffic regime $\lambda=0.1,0.4,0.8$ given $\mathrm{MAC}$ parameters $m_{0}=3, m=4, m_{b}=8, n=1$, the number of devices $N=10$, and packet length $L=7$ in the simulation.

analyzed. A false negative failure is that carrier sensing incorrectly detects that the medium is idle when it is actually busy. A false positive event occurs when a busy state is reported when the medium is idle. We model carrier sensing failures by independent Bernoulli trials with success probability $1-\gamma$ where $0<\gamma<1$. The independence of the trial results is assumed to be over all links and time slots. 


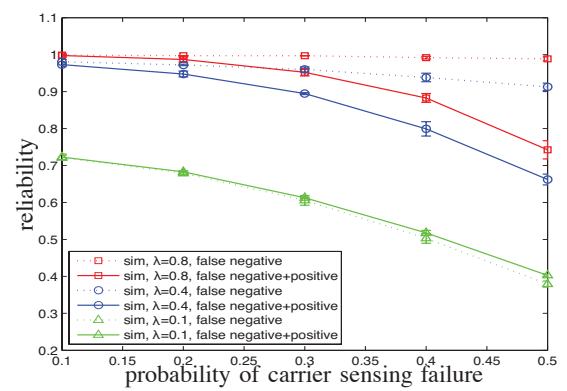

(a) Reliability

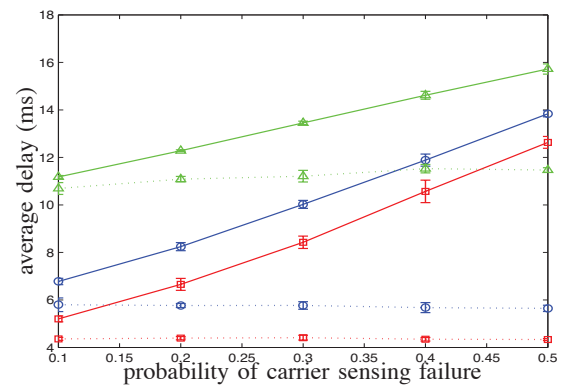

(b) Average delay

Fig. 10: Effect of imperfect carrier sensing as a function of false probabilities $\gamma=0.1, \ldots, 0.5$, traffic regime $\lambda=0.1,0.4,0.8$ in the simulation.

Fig. 10 presents the reliability and average packet delay of simulation results as a function of the probabilities of the carrier sensing error $\gamma=0.1, \ldots, 0.5$, traffic regime $\lambda=$ $0.1,0.4,0.8$ given MAC parameters $m_{0}=3, m=4, m_{b}=$ $8, n=1$, the number of devices $N=10$, and packet length $L=7$. Note that "false negative" and "false negative+positive" refer to the consideration of the false negative event and the combined event of the false negative and positive failure for the simulation setup, respectively. The false negative and positive failure decreases the system performance in terms of the reliability and average packet delay. The false negative failure is critical because a device sends a packet even if the medium is actually busy. In [38], the authors show that the false positive failure is irrelevant to achievable throughput. However, we remark that this observation does not hold if the number of backoffs is limited and the traffic load is low. The false positive failure significantly decreases the performance in terms of the reliability and average packet delay for the lower traffic regime $\lambda=0.4,0.8$ due to the limited number of backoffs. The busy channel probability increases as the false positive failure increases. However, the false positive failure has negligible effects on the reliability of the higher traffic load $\lambda=0.1$. The false positive failures tend to defer the transmission and in fact are beneficial to compensate the effect of the false negative failures. Because false positive failures create extra backoffs, they increase the packet delay.

Fig. 11 illustrates the sensitivity of adaptive IEEE 802.15.4 with respect to the estimation errors to the busy channel probabilities $\alpha$ and $\beta$ and the channel access probability $\tau$. The normalized root mean squared deviation (NRMSD) between the optimal MAC parameters with exact estimation and the ones with erroneous estimation is used as the indicator of sensitivity. The normalization is taken over the range of MAC parameters $\left(m_{0}, m, n\right)$. The NRMSD is approximately below

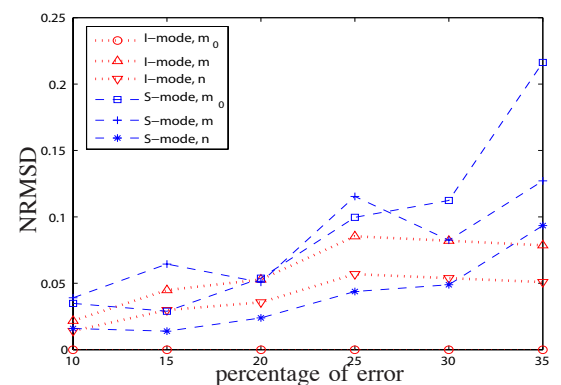

Fig. 11: Sensitivity: NRMSD of $I$-mode and $S$-mode when the traffic load $\lambda=0.6$, length of the packet $L=3$, reliability requirement $R_{\min }=0.95$ and delay requirement $D_{\max }=100 \mathrm{~ms}$, and $N=10$ devices with different percentage error in busy channel probabilities and channel access probability in the simulation.

$10 \%$ if the percentage of error is smaller than $20 \%$ for $\alpha, \beta, \tau$. It is interesting to observe that $m_{0}$ of $I$-mode is very robust to errors. This is due to the power consumption model, i.e., to the dominant factor $m_{0}$ of power consumption in I-mode. The robustness of MAC parameter is $m_{0}>n>m$ and $n>m>m_{0}$ for $I$-mode and $S$-mode, respectively. We can show that errors below $20 \%$ in the estimation of $\alpha, \beta, \tau$ give a performance degradation below $3 \%$ in terms of reliability, packet delay and energy gain for low traffic load.

\section{Conclusions}

In this paper we developed an analysis based on a Markov chain model of IEEE 802.15.4, including retry limits, ACK and unsaturated traffic regime. Then, we presented an adaptive MAC algorithm for minimizing the power consumption while guaranteeing reliability and delay constraints of the IEEE 802.15.4 protocol. We provided a test-bed implementation of the protocol, building a WSN with TelosB sensors and Contiki OS. We investigated the performance of our algorithm under both stationary and transient conditions by experiments and Monte Carlo simulations. Numerical results showed that the proposed scheme is efficient and ensures a longer lifetime of the network. In addition, we showed that, even if the number of active devices and traffic configuration change sharply, our algorithm allows the system to recover quickly and operate at its optimal parameter by estimating just the busy channel and channel access probabilities. We also studied the robustness of the protocol to possible errors during the estimation process on number of devices and traffic load. Results indicated that the protocol reacts promptly to erroneous estimations. Furthermore, we showed that the effect of imperfect channel and carrier sensing failures on system performance heavily depends on the traffic load and limited range of the protocol parameters. The false positive failure affects not only the packet delay but also the reliability in the lower traffic load mainly due to the limited number of backoffs.

\section{References}

[1] A. Nayak and I. Stojmenovic, Wireless Sensor and Actuator Networks. John Wiley \& Sons, 2010.

[2] J. P. Hespanha, P. Naghshtabrizi, and Y. Xu, "A survey of recent results in networked control systems," Proceedings of the IEEE, vol. 95, no. 1, pp. 138-162, 2007.

[3] IEEE 802.15.4 standard: Wireless Medium Access Control and Physical Layer Specifications for Low-Rate Wireless Personal Area Networks, IEEE, 2006, http://www.ieee802.org/15/pub/TG4.html. 
[4] S. Pollin, M. Ergen, S. C. Ergen, B. Bougard, L. Perre, I. Moerman, A. Bahai, P. Varaiya, and F. Catthoor, "Performance analysis of slotted carrier sense IEEE 802.15.4 medium access layer,' IEEE Transactions on Wireless Communication, vol. 7, no. 9, pp. 3359-3371, 2008.

[5] IEEE 802.15 task group 4e: Wireless Medium Access Control and Physical Layer Specifications for Low-Rate Wireless Personal Area Networks, IEEE, 2010, http://www.ieee82.org/15/pub/TG4e.html.

[6] Enocean Products, Enocean Alliance, http://www.enocean.com/.

[7] IEEE 802.11 standard: Wireless LAN Medium Access Control and Physical Layer Specifications, IEEE, 1999, http://www.ieee802.org/11.

[8] G. Bianchi, "Performance analysis of the IEEE 802.11 distributed coordination function," IEEE Journal on Selected Areas in Communications, vol. 18, no. 3, pp. 535-547, 2000.

[9] O. Tickioo and B. Sikdar, "Queueing analysis and delay mitigation in IEEE 802.11 random access MAC based wireless networks," in IEEE INFOCOM, 2004

[10] H. Wu, Y. Peng, K. Long, S. Cheng, and J. Ma, "Performance of reliable transport protocol over IEEE 802.11 wireless LAN: Analysis and enhancement," in IEEE INFOCOM, 2002.

[11] J. Zheng and M. L. Lee, "Will IEEE 802.15.4 make ubiquitous networking a reality?: A discussion on a potential low power, low bit rate standard," IEEE Communications Magazine, vol. 42, no. 6, pp. 140-146, 2004.

[12] A. Faridi, M. Palattella, A. Lozano, M. Dohler, G. Boggia, L. Grieco, and P. Camarda, "Comprehensive evaluation of the IEEE 802.15.4 MAC layer performance with retransmissions," IEEE Transactions on Vehicular Technology, vol. 59, no. 8, pp. 3917-3932, 2010.

[13] C. Y. Jung, H. Y. Hwang, D. K. Sung, and G. U. Hwang, "Enhanced Markov chain model and throughput analysis of the slotted CSMA/CA for IEEE 802.15.4 under unsaturated traffic conditions," IEEE Transactions on Vehicular Technology, vol. 58, no. 1, pp. 473-478, 2009.

[14] J. Misic, S. Shaf, and V. B. Misic, "Performance of a beacon enabled IEEE 802.15.4 cluster with downlink and uplink traffic," IEEE Transactions on Parallel and Distributed Systems, vol. 17, no. 4, pp. 361-376, 2006.

[15] C. Buratti, "Performance analysis of IEEE 802.15.4 beacon-enabled mode," IEEE Transactions on Vehicular Technology, vol. 59, no. 4, pp. 2031-2045, 2010

[16] X. Ling, Y. Cheng, J. W. Mark, and X. Shen, "A renewal theory based analytical model for the contention access period of IEEE 802.15.4 MAC," IEEE Transactions on Wireless Communications, vol. 7, no. 6 , pp. 2340-2349, 2008.

[17] Y.-K. Huang, A.-C. Pang, and H.-N. Hung, "A comprehensive analysis of low-power operation for beacon-enabled IEEE 802.15.4 wireless networks," IEEE Transactions on Wireless Communications, vol. 8, no. 11, pp. 5601-5611, 2009.

[18] A. Giridhar and P. R. Kumar, "Toward a theory of in-network computation in wireless sensor networks," IEEE Communication Magazine, pp. 97-107, 2006

[19] TelosB Device, Crossbow, http://www.xbow.com/.

[20] R. Cristescu, B. Beferull-Lozano, M. Vetterli, and R. Wattenhofer, "Network correlated data gathering with explicit communication: NPcompleteness and algorithms," IEEE/ACM Transactions on Networking, vol. 14 , no. 1 , pp. 41-54, 2006.

[21] F. Cali, M. Conti, and E. Gregori, "IEEE 802.11 protocol: design and performance evaluation of an adaptive backoff mechanism," IEEE Journal on Selected Areas in Communications, vol. 18, no. 9, pp. 1774 $-1786,2000$.

[22] R. Bruno, M. Conti, and E. Gregori, "Optimization of efficiency and energy consumption in p-persistent CSMA-based wireless LANs," IEEE Transactions on Mobile Computing, vol. 1, no. 1, pp. 10 - 31, 2002.

[23] K. Yedavalli and B. Krishnamachari, "Enhancement of the IEEE 802.15.4 MAC protocol for scalable data collection in dense sensor networks," in ICST WiOPT, 2008.

[24] A. Nafaa and A. Ksentini, "On sustained QoS guarantees in operated IEEE 802.11 wireless lans," IEEE Transactions on Parallel and Distributed Systems, vol. 19, no. 8, pp. 1020 -1033, 2008.

[25] V. Bharghavan, A. J. Demers, S. Shenker, and L. Zhang, "MACAW: A media access protocol for wireless LAN's," in ACM SIGCOMM, 1994.

[26] B. Bensaou, Y. Wang, and C. C. Ko, "Fair medium access in 802.11 based wireless ad-hoc networks," in ACM MobiHoc, 2000.

[27] P. Patras, A. Banchs, P. Serrano, and A. Azcorra, "A control-theoretic approach to distributed optimal configuration of 802.11 WLANs," IEEE Transactions on Mobile Computing, vol. 10, no. 6, pp. 897-910, 2011.

[28] J. Ma, M. Gao, Q. Zhang, and L. Ni, "Energy-efficient localized topology control algorithms in IEEE 802.15.4-based sensor networks," IEEE
Transactions on Parallel and Distributed Systems, vol. 18, no. 5, pp. 711-720, 2007.

[29] J. Misic, S. Shafi, and V. Misic, "Maintaining reliability through activity management in an 802.15.4 sensor cluster," IEEE Transactions on Vehicular Technology, vol. 55, no. 3, pp. 779 - 788, 2006.

[30] P. Park, C. Fischione, and K. H. Johansson, "Adaptive IEEE 802.15.4 protocol for energy efficient, reliable and timely communications," in ACM/IEEE IPSN, 2010

[31] P. Park, P. D. Marco, P. Soldati, C. Fischione, and K. H. Johansson, "A generalized Markov chain model for effective analysis of slotted IEEE 802.15.4," in IEEE MASS, 2009.

[32] F. Osterlind and A. Dunkels, "Approaching the maximum 802.15.4 multi-hop throughput," in ACM HotEmNets, 2008.

[33] P. Park, P. D. Marco, C. Fischione, and K. H. Johansson, "Adaptive IEEE 802.15.4 protocol for reliable and timely communications," KTH, Tech. Rep., 2010, http://www.ee.kth.se/ pgpark/papers/ TRITA-EE-201110.pdf.

[34] D. P. Bertsekas and J. N. Tsitsiklis, Parallel and Distributed Computation: Numerical Methods. Athena Scientific, 1997.

[35] F. Cali, M. Conti, and E. Gregori, "Dynamic tuning of the IEEE 802.11 protocol to achieve a theoretical throughput limit," IEEE/ACM Transactions on Networking, vol. 8, no. 6, pp. 785 - 799, 2006.

[36] A. Dunkels, B. Gronvall, and T. Voigt, "Contiki - a lightweight and flexible operating system for tiny networked sensors," in IEEE EmNets, 2004.

[37] Y. Qin and P. Park, "Implementation of adaptive IEEE 802.15.4 protocol," KTH, Tech. Rep., 2010, [Online] Available: http://www.ee.kth.se/ $\sim$ pgpark/code/adap-wpan.zip.

[38] T. H. Kim, J. Ni, R. Srikant, and N. H. Vaidya, "On the achievable throughput of CSMA under imperfect carrier sensing," in IEEE INFOCOM, 2011.

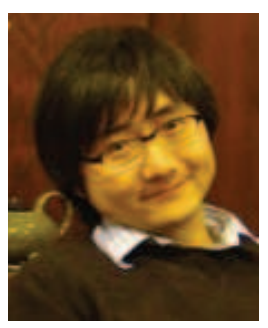

Pangun Park received the M.S. and Ph.D. degrees in electrical engineering from the Royal Institute of Technology, Sweden, in 2007 and 2011, respectively. Since 2011, he has been working as a postdoctoral researcher in electrical engineering and computer science from the University of California, Berkeley. His research interests include cyber-physical systems, and wireless sensor and actuator networks.

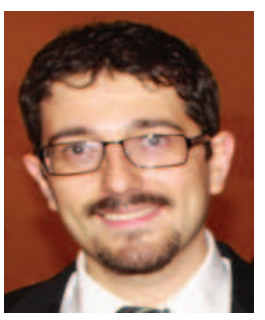

Piergiuseppe Di Marco received the M.Sc. degree in telecommunications engineering from the University of L'Aquila, Italy, in 2008. He is currently working towards the Ph.D. degree in telecommunications at the Automatic Control Laboratory, School of Electrical Engineering, Royal Institute of Technology (KTH), Stockholm, Sweden. His research interests include modeling, design, and optimization for wireless sensor networks.

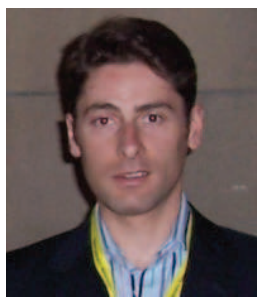

Carlo Fischione is a tenured Associate Professor at KTH Royal Institute of Technology, Electrical Engineering, Stockholm, Sweden. His research interests include optimization and parallel computation with applications to wireless sensor networks, networked control systems, and wireless networks. He received the best paper award from the IEEE Transactions on Industrial Informatics of 2007. He is Member of IEEE and SIAM.

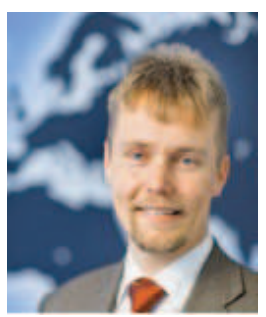

Karl H. Johansson is Director of the KTH ACCESS Linnaeus Centre and Professor at the School of Electrical Engineering, Royal Institute of Technology, Sweden. He is a Wallenberg Scholar, has held a Senior Researcher Position with the Swedish Research Council, and visiting positions at UC Berkeley and California Institute of Technology. His research interests are in networked control systems, hybrid and embedded control, and control applications in automotive, automation and communication systems. He was a member of the IEEE Control Systems Society Board of Governors 2009, the Chair of the IFAC Technical Committee on Networked Systems 2008-2011, and has been on the Editorial Boards of IEEE Transactions on Automatic Control and the IFAC journal Automatica. 